\title{
NON-INVASIVE PREDICTORS FOR THE PRESENCE, GRADE AND RISK OF BLEEDING FROM ESOPHAGEAL VARICES IN PATIENTS WITH POST-HEPATITIC CIRRHOSIS
}

\author{
By
}

Ahmed El Ray ${ }^{1}$, Mohamed Mohamed Azab ${ }^{2}$, Ibrahim Mohamed Abd El-Aziz ${ }^{2}$, Ahmed Abd El-Aleem², Mohamed Darwish El-Talkawy", Mohamed Abd El-Hameed Abd El-Badea*, Mahmoud El Ansary ${ }^{1}$, Abdelaziz Ali Saleem ${ }^{1}$ and Tarek Mahmoud Diab ${ }^{3}$.

Department of Hepatogastroenterology ${ }^{1}$, and Department of Tropical Medicine ${ }^{2}$, Faculty of Medicine, Al-Azhar University, Cairo, Department of Parasitology ${ }^{3}$, Theodor Bilharz Research Institute ${ }^{1,3}$, Imbaba P. O. Box 30, Giza, Egypt.

\begin{abstract}
Variceal bleeding is the last step of a chain of events initiatedby an increase in portal pressure, followed by the development and progressive dilation ofvarices until these finally rupture and bleed. The ideal method to diagnose portal hypertension should be accurate, noninvasive, objective, and reproducible. The study evaluated the predictive value of two non-invasive parameters for the diagnosis of esophageal varices (EV): 1- Right liver lobe diameter/serum albumin ratios (RLLD /S. albumin), and 2- Platelet count/splenic bipolar diameter ratios (Platelets count /SBPD). This study included eighty Egyptian patients with post-hepatitic cirrhosis (45males and 35 females). They underwent laboratory ultrasono-graphic and endoscopic examinations within one week. RLLD/S. albumin and Platelets count/SBPD ratios were calculated. The results showed that EV were not detected by upper digestive endoscopy in $25 \%$, while grade I of EV was found in $17.5 \%$, grade II in $17.5 \%$, grade III in $20 \%$, \& grade IV in $20 \%$. RLLD/S. albumin concentration ratio diagnosed the varices at cut off value of 3.43 with $95 \%$ sensitivity and $80 \%$ specificity. Also, it was positively correlated with grading of E.V, when this ratio increased the grading of E.V increases and vice versa. Besides, it predicted bleeding from E.V. at cut off value of 5.096 with $63 \%$ sensitivity and $73 \%$ specificity. Platelet count/ SBPD ratio predicted the presence of varices at cut off value 1847 with $95 \%$ sensitivity and $93 \%$ specificity, and negatively correlated with grading of $\mathrm{EV}$, when this ratio decreased grading of E.V increase and vice versa. It also predicted bleeding from E.V. at cut off value of 4809 with $50 \%$ sensitivity and $93 \%$ specificity.
\end{abstract}

Key words: Right liver lobe diameter, esophageal varices, platelet count, splenic bipolardiameter.

\section{Introduction}

Variceal bleeding is the last step of a chain of events initiated by an increase in portalpressure, followed by the development and progressive dilation of varices until these finally rupture and bleed. The appearance of varices incompensated patients indicates a change from a clinical stage with a very low risk of death at 1 year (stage $1 ; 1 \%$ risk) to an intermediate risk stage (stage $2 ; 3.4 \%$ risk). The occurrence of variceal bleeding isa dreadful event, marking the progression to decompensation of the liver disease and to a very high risk of death stage; stage $4 ; 57 \%$ risk (D'Amico, 2006).

Esophagogastroduodenoscopy (EGD), via invasive, remained the gold standard for screening of esophageal varices in cirrhotic patients over many years. EGD directly visualizes esophageal varices and signs of impending rupture (Garcia-Tsao, 2001). The ideal method to diagnose portal hypertension should be accurate, noninvasive, objective and reproducible. Existing methods for diagnosis of portal hypertension includeclinical examination, laboratory tests, imaging studies, HVPG measurement and EGD. Some of those methods are invasive, while others are noninvasive.

Noninvasive biochemical prediction of EV was the subject of several previous studies. Assessed biochemical parameters included hemoglobin, platelet count, leukocyte count, serum albumin, and serum bilirubin and pro- 
thrombin time in relation to esophageal varices Giannini et al. (2003). Clinical prediction of EV through medical history and thorough clinical examination has been the earliest and cheapest method of diagnosis of portal hypertension and its complications. Ascites, splenomegaly, shrunken liver and hepatic encephalopathy are directly correlated to the presence of esophageal varices (Schepis et al, 2001).

Several ratios combining radiological and biochemical parameters have been introduced for prediction of presence of EV. Those ratios included Platelet count/ splenic bipolar diameter (SBPD) ratio and Right liver lobe diameter (RLLD)/ Serum albumin ratio. Sensitivity of Platelet count/ SBPD ratio in predicting the presence of esophageal varices was $100 \%$ and the specificity was $93 \%$. Sensitivity of RLLD/ Serum albumin concentration ratio in prediction of presence of esophageal varices was $83.1 \%$ and the specificity was $73.9 \%$ (Alempijevic et al, 2007).

Routine endoscopic screening of all patients with liver cirrhosis with or without EV has health service cost implications, as $35 \%$ to $70 \%$ of patients with cirrhosis have EV and up to $30 \%$ have large varices. Therefore, it might be cost-effective to identify those patients who would benefit most from routine screening (Giannini et al, 2003).

This work aimed to evaluate the predictive value of 2 non-invasive parameters (RLLD/ serum albumin ratios and Platelet count/ SBPD ratios) for the diagnosis of EV; their grading and the prediction of bleeding varices.

\section{Patients, Materials and Methods}

This study includes 80 patients treated for post viral liver cirrhosis admitted to the department of Hepatogastroenterology in Theodor Bilharz Research Institute from July 2010 to June 2011. Cirrhosis diagnosis was based on clinical features, laboratory test, imaging diagnostics, and liver histology whenever possible.

The following information was collected for each patient: age, gender, etiology of cir- rhosis, biochemical parameters (aspartate aminotransferase, alanine aminotransferase, total bilirubin, serum albumin, prothrombin activity, and platelet count), presence and degree of esophageal varices and degree of liver function Impairment by Child-Pugh classes. Cirrhosis etiology was determined as viral if hepatitis B surface antigen or hepatitis $\mathrm{C}$ serum markers were positive. All studied subjects underwent ultrasonographic examination of the upper abdomen. The right lobe diameters (RLLD) in the mid clavicular line, as well as the spleen bipolar diameter (SBPD) were measured for three times and the mean value was recorded.

Using the laboratory and ultrasonographic values, the study calculated two ratios: RLLD/S.albumin and RLLD /S.albumin and Platelets count /SBPD. Varices in the level of mucosa were recognized as grade I, those smaller than $5 \mathrm{~mm}$ fulfilling less than $1 / 3$ of the esophageal lumen were recognized as grade II , grade III were varices larger than 5 $\mathrm{mm}$ fulfilling more than $1 / 3$ of the esophageal, while grade IV varices occupied more than $2 / 3$ of esophageal lumen. Patients with previous variceal bleeding, porto-systemic shunts and those taking beta blockers medications and patients with coexistent illness or infection that could influence the liver and spleen size were excluded.

Child-Pugh score was calculated using five variables (ascites, encephalopathy, bilirubin, albumin, and prothrombin time). Values 1, 2 or 3 were assigned to each of these variables due to increasing abnormality, and the score calculated as sum of the five variables for each patient. A Child-Pugh score less than 7 was considered as class A, from 7 to 9 as class B, while any score greater than 9 was as class C. Laboratory test, ultrasonographic and endoscopic examinations were performed within one week.

The Ethic Committee of Theodor Bilharz Research Institute approved the study (FWA 00010609.) and all patients were given an informed consent prior to inclusion. 
Statistical analysis was performed using Statistical Package for Social Sciences (SPSS, version 10.0). Basic descriptive statistics included means, standard deviations, ranges and percentages. For correlation analysis, we used Spearman's test. Differences were considered statistically significant if the two-tailed $\mathrm{P}$ value was less than 0.05 . Sensitivity and specificity, as well as the best cut-off value for the diagnosis of varices were calculated using ROC curves.

\section{Results}

According to upper GIT endoscopic findings, the patients were classified into 5 groups:

Table 1: Demographic features of groups

\begin{tabular}{|c|c|c|c|c|c|}
\hline Items & $\mathrm{G} 1(\mathrm{n}=20)$ & $\mathrm{G} 2(\mathrm{n}=14)$ & $\mathrm{G} 3(\mathrm{n}=14)$ & $\mathrm{G} 4(\mathrm{n}=16)$ & $\mathrm{G} 5(\mathrm{n}=16)$ \\
\hline Age $(\mathrm{yrs})$. & $39-63$ & $34-67$ & $34-67$ & $43-66$ & $45-67$ \\
\hline Mean \pm SD & $46.70 \pm 6.53$ & $52.50 \pm 8.64$ & $52.43 \pm 10.86$ & $54.13 \pm 6.78$ & $54.94 \pm 6.37$ \\
\hline \multirow{2}{*}{ Female/Male } & $11 / 9$ & $8 / 6$ & $6 / 8$ & $4 / 12$ & $6 / 10$ \\
& $(55 / 45 \%)$ & $(57.14 / 42.86 \%)$ & $(42.86 / 57.14 \%)$ & $(25 / 75 \%)$ & $(37.5 / 62.5 \%)$ \\
\hline
\end{tabular}

Table 2: Child's classification in the studied groups

\begin{tabular}{|c|c|c|c|}
\hline Items & Child A & Child B & Child C \\
\hline G1 $(n=20)$ & $19(95 \%)$ & $1(5 \%)$ & $0(0 \%)$ \\
\hline G2 $(n=14)$ & $7(50 \%)$ & $4(28.6 \%)$ & $3(21.4 \%)$ \\
\hline G3 $(n=14)$ & $1(7.1 \%)$ & $9(64.3 \%)$ & $4(28.6 \%)$ \\
\hline G4 $(n=16)$ & $1(6.3 \%)$ & $5(31.3 \%)$ & $10(62.5 \%)$ \\
\hline G5 $(n=16)$ & $1(6.3 \%)$ & $4(25 \%)$ & $11(68.8 \%)$ \\
\hline
\end{tabular}

RLLD/S. albumin ratio, Platelet count/ SBPD ratio, SBPD and S.albumin were significant in differentiation between patients without varices and patients with varices; RLLD showed non-significant in difference between patients with or without varices.

Table 3: Comparison of calculated variables in patients with and without esophageal varices.

\begin{tabular}{|l|c|c|c|}
\hline Items & No EV $(\mathrm{n}=20)$ & $\mathrm{EV}(\mathrm{n}=60)$ & $p$ - value \\
\hline RLLD /S. albumin & $3.94 \pm 0.88$ & $5.28 \pm 1.86$ & $0.003^{* *}$ \\
\hline Platelets count /SBPD & $15054.46 \pm 4206.85$ & $6613.56 \pm 3468.46$ & $0.001^{* *}$ \\
\hline S. albumin & $3.57 \pm 0.47$ & $2.57 \pm 0.64$ & $0.001^{* *}$ \\
\hline RLLD & $13.89 \pm 2.40$ & $12.71 \pm 2.74$ & 0.089 \\
\hline Platelets count & $184350.00 \pm 47942.15$ & $100298.33 \pm 44613.76$ & $0.001^{* *}$ \\
\hline SBPD & $12.56 \pm 1.57$ & $15.46 \pm 2.13$ & $0.001^{* *}$ \\
\hline
\end{tabular}

Values $=$ mean $\pm \mathrm{SD} .{ }^{* *} p<0.01=$ highly significant.

All patients, RLLD/S.albumin ratio showed positive significant correlations with E.V. i.e., any decrease in RLLD/ S.albumin ratio was possibly associated with a decrease in grading of EV and Child's score. Platelets

count/SBPD ratio showed significant negative correlation with EV \& Child's score i.e., any decrease in that ratio (as in advanced cirrhosis) was associated with increase in grading of EV and Child's score (Tab.4).

Table 4: Correlation of RLLD /S.albumin and Platelets count/SBPD ratios with EV and Child's score

\begin{tabular}{|c|c|c|c|c|}
\hline Variable & \multicolumn{2}{|c|}{ RLLD/S.albumin ratio } & \multicolumn{2}{c|}{ Platelets count /SBPD Ratio } \\
\hline Correlated & $\mathrm{r}$ & $P$ & $\mathrm{R}$ & $p$ \\
\hline EV & 0.305 & $0.018^{*}$ & -0.633 & $0.001^{* *}$ \\
\hline Child's score & 0.316 & $0.014^{*}$ & -0.399 & $0.002^{* *}$ \\
\hline
\end{tabular}




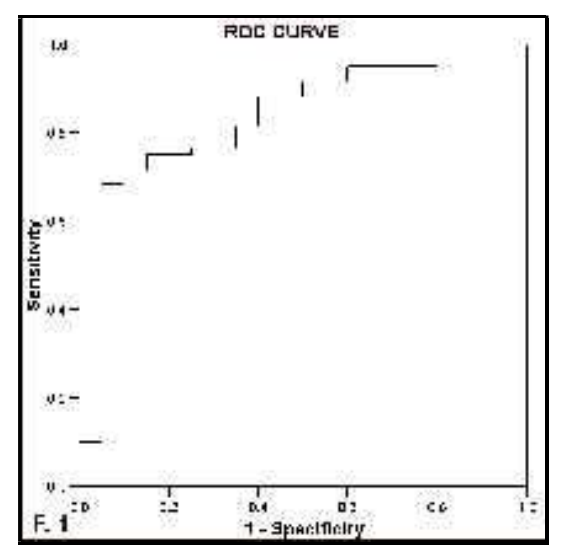

Fig. 1: ROC curve showed cut off value for best sensitivity and specificity of RLLD /S.albumin ratio in difference between patients with and without EV. AUC $=0.834$ with best cut off value at 3.34 where sensitivity was $95 \%$ \& specificity $80 \%$.

Test Result Variable(s): RLLD/S.albumin

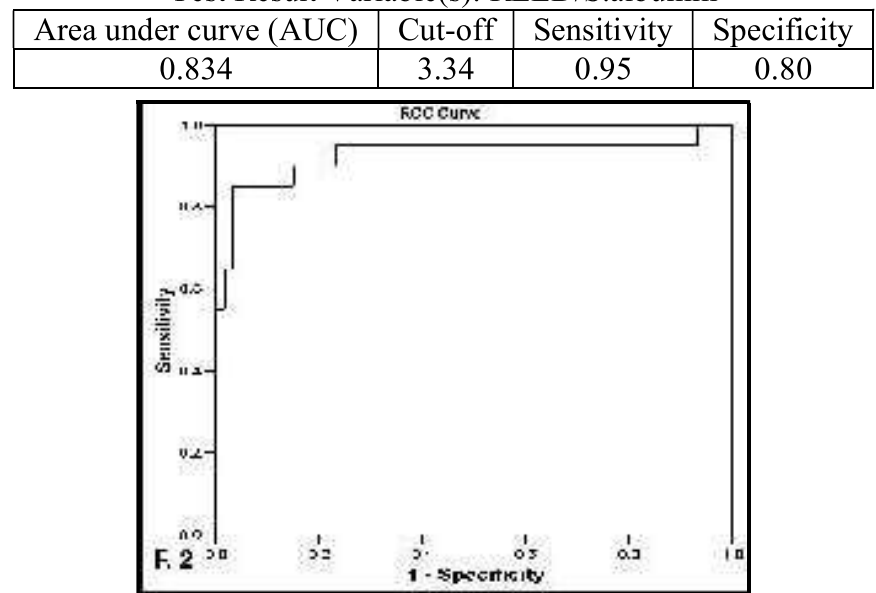

Fig. 2: ROC curve showed cut off value for best sensitivity and specificity of Platelets count /SBPD ratio differentiated between patients with or without varices. Platelet count/SBPD ratio proved highly sensitive and less specific in differentiation between patients with and without esophageal varices with best cut off value at 1847 . AUC $=0.926$ where sensitivity $95 \% \&$ specificity $93 \%$.

\begin{tabular}{|l|l|l|l|}
\hline Area under curve (AUC) & Cut-off & Sensitivity & Specificity \\
\hline 0.926 & 1847 & 0.95 & 0.93 \\
\hline
\end{tabular}

Table 5: Ratios and ultrasonographic findings in groups.

\begin{tabular}{|l|c|c|c|c|c|c|}
\hline Variables & Control $(\mathrm{n}=20)$ & $\mathrm{G} 1(\mathrm{n}=14)$ & $\mathrm{G} 2(\mathrm{n}=14)$ & $\mathrm{G} 3(\mathrm{n}=16)$ & $\mathrm{G} 4(\mathrm{n}=16)$ & $p$-value \\
\hline Platelets count/SBPD & $15054.46 \pm$ & $9334.85 \pm$ & $8263.31 \pm$ & $5688.97 \pm$ & $3713.49 \pm$ & \multirow{2}{*}{$0.001^{* *}$} \\
Ratio & 4206.85 & 2103.87 & 3053.18 & 3335.42 & 2223.43 & \\
\hline RLLD/S. albumin ratio & $3.94 \pm 0.88$ & $4.52 \pm 0.91$ & $4.94 \pm 1.07$ & $5.53 \pm 1.48$ & $6.35 \pm 2.42$ & $0.001^{* *}$ \\
\hline Splenic diameter $(\mathrm{cm})$ & $12.56 \pm 1.57$ & $13.84 \pm 1.33$ & $14.36 \pm 0.91$ & $16.09 \pm 1.82$ & $17.21 \pm 2.23$ & $0.001^{* *}$ \\
\hline Rt. Lobe diameter $(\mathrm{cm})$ & $13.89 \pm 2.40$ & $13.89 \pm 1.88$ & $13.54 \pm 2.26$ & $12.49 \pm 1.96$ & $12.15 \pm 2.4$ & $0.074(\mathrm{NS})$ \\
\hline PV diameter $(\mathrm{cm})$ & $11.39 \pm 1.28$ & $12.42 \pm 1.53$ & $13.67 \pm 1.24$ & $15.06 \pm 0.90$ & $15.83 \pm 1.74$ & $0.001^{* *}$ \\
\hline
\end{tabular}

Table 6: Ratios and ultrasonographic findings in control, esophageal varices without bleeding (GA) and bleeding esophageal varices (GB)

\begin{tabular}{|l|c|c|c|c|}
\hline Variables & Control $(\mathrm{n}=20)$ & GA $(\mathrm{n}=38)$ & GB $(\mathrm{n}=22)$ & $p$-value \\
\hline platelets count/SBPD Ratio & $15054.46 \pm 4206.84$ & $7606.51 \pm 2791.98$ & $5620.61 \pm 3826.24$ & $0.001^{* *}$ \\
\hline RLLD / S.albumin Ratio & $3.94 \pm 0.88$ & $4.78 \pm 1.16$ & $5.78 \pm 2.27$ & $0.001^{* *}$ \\
\hline Splenic diameter & $12.56 \pm 1.57$ & $14.60 \pm 1.51$ & $16.32 \pm 2.33$ & $0.001^{* *}$ \\
\hline Rt. Lobe diameter & $13.89 \pm 2.40$ & $13.20 \pm 1.87$ & $12.21 \pm 3.36$ & 0.085 \\
\hline PV diameter & $11.39 \pm 1.28$ & $13.21 \pm 1.57$ & $15.44 \pm 1.90$ & $0.001^{* *}$ \\
\hline
\end{tabular}

$p$-value by ANOVA test. $* * p<0.01=$ highly significant.

\begin{tabular}{|l|l|l|l|}
\hline Area under curve (AUC) & Cut-off & Sensitivity & Specificity \\
\hline 0.671 & 5.096 & 0.63 & 0.73 \\
\hline
\end{tabular}




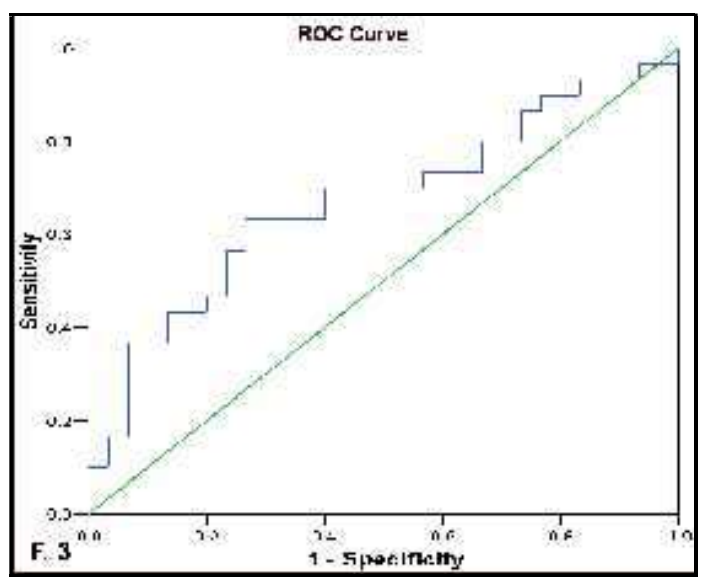

Fig. 3: A ROC curve to determine cut off value for best sensitivity and specificity of RLLD /S.albumin ratio in differentiation between esophageal varices without bleeding and bleeding varices.

RLLD/S. albumin ratio was sensitive and specific to off value at 5.096 where sensitivity $63 \% \&$ specificidistinguish between esophageal varices in patients ty $73 \%$.

with or without bleeding. (AUC=0.671) with best cut

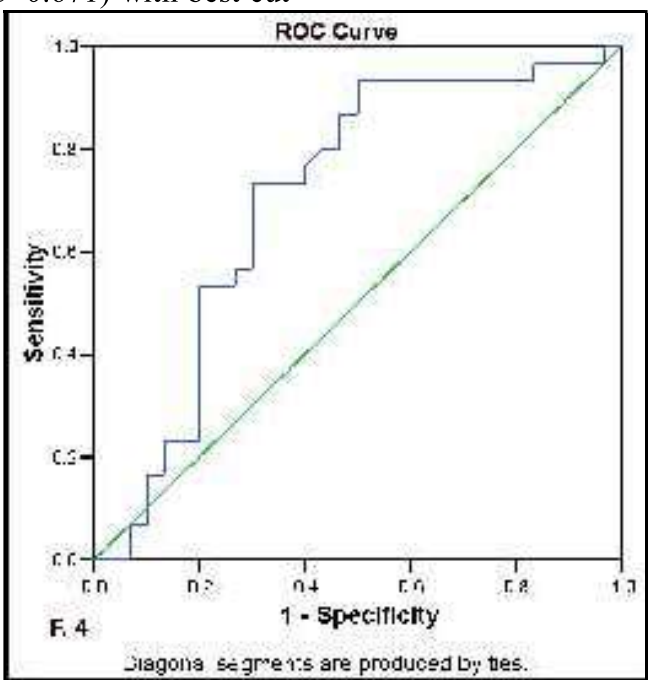

Figure 4: ROC curve to determine cut off value for best sensitivity and specificity of platelet count/SBPD ratio in differentiation between esophageal varices without bleeding and patients with bleeding varices.

\begin{tabular}{|l|l|l|l|}
\hline Area under curve (AUC) & Cut-off & Sensitivity & Specificity \\
\hline 0.706 & 4809 & 0.50 & 0.93 \\
\hline
\end{tabular}

Platelet count/SBPD ratio less sensitive and highly specific to differentiate between patients with or without varices bleeding $(\mathrm{AUC}=0.706$.) with best cut off value at 4809 , sensitivity $50 \%$ and specificity $93 \%$.

\section{Discussion}

It was estimated that 100 screening endoscopic examinations need to be performed to prevent 1 to 2 cases of variceal bleeding. Therefore, the identification of clinical features and investigations that can accurately predict EV and help identifying patients at greatest risk are important. Predictors of bleeding should help to identify patients with the highest prevalence of esophageal varices and improve the yield and cost- effectiveness of endoscopic screening (Berzigotti et al, 2011). Repeated endoscopic examinations are unpleasant for patients, and have cost impact on health care insurance. Therefore, numerous non-invasive parameters have been investigated for assessment of presence of esophageal varices, its grading, and prediction for bleeding (GarciaTsao, 2001).

In the present cohort $\mathrm{S}$. albumin showed highly significant differences $(\mathrm{P}<0.01)$ in 
patients with EV compared to patients without varices. These results agreed with Sharma et al. (2007) who found that S. albumin was lowest in patients with large esophageal varices with a mean value $=3.0$, range $=1.9$ 4.6, while in patients without esophageal varices, S. albumin was higher with a mean value of $=3.2$, range $=1.6-4.5$. The present results could be explained by the fact that the rate of albumin synthesis was reduced up to $50 \%$ in chronic liver disease and S. albumin was reduced in cirrhosis due to an elevated distribution volume in haemodilution, particularly in association with ascites. Hypoalbuminemia is considered a very important marker of liver dysfunction. However, hypoalbuminemia with chronic liver disease can complicate several associated conditions such as poor nutritional status, immune mediated nephritis associated with viral hepatitis, protein losing enteropathies and any associated inflammatory process. Therefore, hypoalbuminemia may not be the result of liver dysfunction alone. And that is why; it could not stand alone as a single parameter for prediction of presence and risk of bleeding of esophageal varices. However, it has been used in relation to right liver lobe diameter as another marker for liver dysfunction to predict varices (Garcia-Tsao, 2001).

RLLD/ S. albumin ratio shows highly significant differences $(\mathrm{P}<0.01)$ in patients with bleeding EV compared to non-bleeding EV and patients without varices. This ratio combined an anatomical and a functional parameter of liver disease. With the progress of liver disease, hepatomegaly was observed, and the synthetic function of the liveris more or less normal. Finally, with further progress of liver disease and occurrence of cirrhosis, the liver becomes shrunken and serum albumin decreases. Although S. albumin level can serve as an index of liver synthetic capacity, several factors make albumin concentrations difficult to interpret and thus affect the ratio. Albumin has a plasma half-life of three weeks; therefore, serum albumin concentrations change slowly in response to alterations in synthesis. Also, because two thirds of the amount of body albumin is located in the extravascular and extracellular space; changes in distribution can alter the serum concentration. Combination of one or more of those factors may alter S. albumin and therefore alter the sensitivity and specificity ratio (Kuntz and Kuntz, 2006).

Statistically, in the present study, RLLD/ S. Albumin ratio proved to be sensitive and specific in differentiation between patients without varices and patients with varices $(\mathrm{AUC}=0.834)$ with the best cut off value at 3.43 where sensitivity was $95 \%$ and specificity was $80 \%$. This agreed with Alempijevic et al. (2007) who found that RLLD/ S.albumin ratio shows overall $83.1 \%$ sensitivity and $73.9 \%$ specificity in the diagnosis of EV at a cut off value of 4.45. Predictive values, likelihood ratios and accuracy of ratio were not calculated (Garcia-Tsao, 2001).

Platelets count was highest in patients without EV compared to patients with EV. Platelet count may decrease for several reasons in chronic liver disease: thrombocytopenia may be secondary to hypersplenism, impaired platelet production, increased fibrinolytic activity, or decreased thrombopoietin production by the diseased liver (Agha et al, 2008). Thus, the use of platelet count alone as a non-invasive predictor of EV can be misleading and cannot be solely attributed to portal hypertension (Zaman, 2003).

The SBPD was highest in patients with bleeding EV compared to non-bleeding EV and patients without EV. The results agreed with Giannini et al. (2003) who found that splenic diameter of patients without EV had a mean value of $110 \mathrm{~mm}$ (range $=90-190$ ) while the splenic diameter of patients with $\mathrm{EV}$ had a mean value of $155 \mathrm{~mm}$ (range $=$ 90-210). Whereas, Agha et al. (2008) found that splenic diameter of patients without EV had a mean value of $123 \mathrm{~mm}$ (range $=98$ 157) and splenic diameter of patients with $\mathrm{EV}$ had a mean value of $141 \mathrm{~mm}$ (range = 102-181). The present results could be explained by higher portal pressure in patients 
with bleeding varices than that of the other two studies. Splenomegaly in chronic liver disease is usually congestive splenomegaly due to portal hypertension (Kuntz and Kuntz, 2006).

Others found no correlation between the splenic size and EV (Pillette et al, 1999; Schepis et al, 2001; Zaman et al, 2001). These differences with the studies that recommend splenic size for the prediction of EV were attributed to differences in the etiology and the stage of liver cirrhosis of the studied population. Thus, using the platelets count/ SBPD ratio in the present study as parameter linking thrombocytopenia to splenic size was taken into consideration the decrease in platelet count which most likely depends on hypersplenism caused by portal hypertension. Platelet count/ SBPD ratio bypasses the possible drawback as it "normalizes" platelet count to splenic sequestration, most likely representing aliquot of thrombocytopenia caused by portal hypertension (Zaman, 2003).

In the present study, platelets count /SBPD ratio was higher in patients without varices, compared to those with non-bleeding or bleeding EV with the best cut off value at 1847 with sensitivity $95 \%$ and specificity $93 \%$. Giannini et al. (2003) found that platelet count/spleen diameter ratio was $100 \%$ sensitivity, $71 \%$ specificity, $96 \%$ positive predictive value $\& 100 \%$ negative predictive value in diagnosis of EV at a cut off value 909 . Giannini et al. (2005) found platelet count/ SBPD ratio $100 \%$ sensitivity, $73 \%$ specificity, $71 \%$ positive predictive value, $100 \%$ negative predictive value and $93.5 \%$ accuracy in diagnosis of EV at a cut off value 909. Agha et al. (2008) found that platelet count/ spleen diameter gave $100 \%$ sensitivity, 97.6\% specificity, $93.8 \%$ positive predictive value, $100 \%$ negative predictive value, 0.01 negative likelihood ratio and 42 positive likelihood ratio for the EV diagnosis in patients with compensated cirrhosis. In decompensated cirrhosis, the ratio showed 100\% sensitivity, $95.9 \%$ specificity, $100 \%$ nega- tive predictive value, $97.6 \%$ positive predictive value, 0.01 negative likelihood ratio and 24.3 positive likelihood ratio for diagnosis of $\mathrm{EV}$ at a cut off value of 909 .

To use these parameters for detection of different grades of $\mathrm{EV}$, the present study found that RLLD had no role in this issue. However, S. albumin concentration, SBPD, RLLD/s. albumin concentration ratio \& platelets count/SBPD ratio gave highly significant values. Serum albumin concentration was lower in patients with higher grade of varices than those with lower grade whatever the history of bleeding or not and RLLD/ serum albumin concentration ratio was significantly increasing in patients with higher grades of varices than those with lower grades. SBPD was higher in patients with higher grade of varices than those with lower grade whatever there a history of bleeding or not and platelets count/SBPD ratio was significantly decreasing in patients with higher grades of varices than those with lower grades. This agreed with Alempijevic et al. (2007) who identified the risk of development of esophageal varices and the parameters grading. Also, to use the previous parameters for prediction of bleeding from EV the present study found RLLD/ S. albumin ratio can predict bleeding at cut off value of 5.096 with $63 \%$ sensitivity and $73 \%$ specificity. Platelets count /SBPD ratio predicted bleeding EV at cut off value of 4809 with $50 \%$ sensitivity and $93 \%$ specificity. Platelet count/SBPD and RLLD/S. albumin ratios gave good predictors for EV (Alempijevic et al, 2007). In the present study, there was also positive significant correlation of $\mathrm{RLLD} / \mathrm{S}$. albumin ratio and negative significant correlation of platelets count /SBPD ratio with the presence of EV. This correlation supports the studies indicated that newly introduced ratio was predictive for EV. Those ratios were less likely to be used to exclude patients from initial endoscopic screening; but it might decrease unnecessary follow up endoscopies and help to identify patients at higher risks for EV development. 
Transient elastography (TE) is a novel non-invasive technology measuring liver stiffness that has gained popularity over the past few years. Although TE has been initially proposed to assess liver fibrosis, a good correlation was reported between liver stiffness values and HVPG as well as presence of EV, suggested that it could be a good tool for noninvasive portal hypertension evaluation (Castera and Bosch, 2012). When HVPG values exceed 10 to $12 \mathrm{~mm} \mathrm{Hg}$, threshold for clinically significant portal hypertension and for the development of varices, portal pressure became largely independent from stiffness/ fibrosis (De Franchis and Dell'Era, 2014). More sophisticated imaging methods were under evaluation as MR Elastography and acoustic radiation force imaging (Berzigotti et al, 2011).

\section{Conclusion}

Combination of different noninvasive tests might provide complementary information, resulting in increased diagnostic value.

The RLLD/S. albumin and platelet count/ SBPD ratios proved as two useful noninvasive parameters. They provided accurate information pertinent to the presence of esophageal varices, their grading and for prediction of bleeding in patients with post-hepatic cirrhosis. The platelet count/spleen diameter ratio might be used for better rationalization of medical resources and use of endoscopy.

\section{References}

Agha, A, Anwar, E, Bashir, K, Savarino, V, Giannini, EG, 2008: External validation of the platelet count/spleen diameter ratio for the diagnosis of esophageal varices in hepatitis $\mathrm{C}$ virusrelated cirrhosis. Dig. Dis. Sci. 54, 3:654-60.

Alempijevic, T, Bulat, V, Djuranovic, S, Kovacevic, $\mathbf{N}$, Jesic, $\mathbf{R}$, et al, 2007: Right liver lobe /albumin ratio: Contribution to non-invasive assessment of portal hypertension. World J. Gastroenterol. 13, 40:5331-5.

Berzigotti, A, Ashkenazi, E, Reverter, E, Abraldes, JG, Bosch, J, 2011: Non-invasive diagnostic and prognostic evaluation of liver cirrhosis and portal hypertension. Dis. Markers 31, 3: 129-38.

Castera, L, Pinzani, M, Bosch, J, 2012: Non- invasive evaluation of portal hypertension by transient elastography. J. Hepatol. 56, 3:696-703.

D'Amico, G, Garcia-Tsao, G, Pagliaro, L, 2006: Natural history and prognostic indicators of survival in cirrhosis: a systematic review of 118 studies. J. Hepatol. 44:217-31.

De Franchis, R, Dell'Era, A, 2014: Invasive and noninvasive methods to diagnose portal hypertension and esophageal varices. Clin. Liver Dis. 18:293-302.

Garcia-Tsao, G, 2001: Current management of the complications of cirrhosis and portal hypertension: Variceal hemorrhage, ascites and spontaneous bacterial peritonitis. Gastroenterol. 120, 3:726-48.

Giannini, E, Botta, F, Borro, P, Risso, D, Romagnoli, $P$, et al, 2003: Platelet count/spleen diameter ratio: proposal and validation of a noninvasive parameter to predict presence of esophageal varices in patients with liver cirrhosis. Gut 52:1200-5.

Giannini, EG, Botta, F, Borro, P, Dulbecco, P, Testa, E, et al, 2005: Application of the platelet count/spleen diameter ratio to rule out the presence of esophageal varices in patients with cirrhosis: a validation study based on follow-up. Dig. Liver Dis. 37, 10:779-85.

Kuntz, E, Kuntz, HD, 2006: Hepatology Principles and Practice, $2^{\text {nd }}$ English Ed., Springer Publications, Germany.

Peck-Radosavljevic, M, 2000: Thrombocytopenia in liver disease. Canad. J. Gastroenterol. 14:D60-6.

Pillette, C, Oberti, F, Aubé, C, Rousselet, M, Bedossa, P, et al, 1999: Non-invasive diagnosis of esophageal varices in chronic liver diseases. J. Hepatol. 31, 5:867-73.

Schepis, F, Cammà, C, Niceforo, D, 2001: Which patients should undergo endoscopic screening for esophageal varices detection? Hepatol. 33:333-8.

Sharma, SK, Aggarwal, R, 2007: Prediction of large esophageal varices in patients with cirrhosis of the liver using clinical, laboratory and imaging parameters. J. Gastroenterol. Hepatol. 22, 11:1909-15.

Zaman, A, 2003: Current management of esophageal varices. Gastroenterol. 6:499-507.

Zaman, A, Decker, T, Lapidus, J, 2001: Risk factors for the presence of varcies in cirrhotic patients without a history of variceal hemorrhage. Arch. Int. Med. 161:2564-70. 